\title{
An ordinal model for analysis of years of education
}

\author{
Benjamin W. Domingue ${ }^{1, \dagger}$, Klint Kanopka ${ }^{1}$, Sam Trejo ${ }^{2,3}$, and Jeremy Freese ${ }^{4}$ \\ ${ }^{1}$ Stanford Graduate School of Education \\ ${ }^{2}$ La Follette School of Public Affairs, University of Wisconsin -Madison \\ ${ }^{3}$ Department of Sociology, University of Wisconsin-Madison \\ ${ }^{4}$ Department of Sociology, Stanford University \\ †ben.domingue@gmail.com
}

\section{Acknowledgements}

The authors would like to thank Elliot Tucker-Drob for his feedback and tweets [https://twitter.com/ tuckerdrob/status/1270475458015113221?s=20]. The HRS (Health and Retirement Study) is sponsored by the National Institute on Aging (grant number NIA U01AG009740) and is conducted by the University of Michigan. 


\begin{abstract}
Years of education is a commonly used outcome variable in many lifecourse studies. We argue that such studies may derive additional insights from analysis of years of education as an ordinal rather than continuous outcome. The conservative approach that we advocate for-the cumulative link modelproduces estimates with acceptable properties when not the true model unlike the standard linear model, which experiences failures when inappropriately used. We illustrate this via simulation. In particular, studies of interactions can yield high levels of false discovery when the linear model is used for analysis of data from the cumulative link model; the cumulative link model avoids this problem. Further, estimates from the cumulative link model can be tested to determine whether the linear model is appropriate. We use empirical data to illustrate additional insights that are readily derived from application of the ordinal model and offer a suggested workflow for future analysis.
\end{abstract}

\title{
1 Introduction
}

Numerous studies focus on years of education (i.e., educational attainment) as a key outcome. Educational attainment is an important variable given that one's level of schooling is a frequent proxy for the culmination of development (and the inputs that affect it) early in the life and a key predictor of job market success, socioeconomic status, and health later in the life course. Given its centrality as an endpoint in understanding child development and well-being and its role as a key link between early-life and later-life, years of education (or a closely-related substitute such as highest degree obtained) is a key variable in fields such as sociology (Herd et al., 2019; Conley et al., 2015; Darnell and Sherkat, 1997; Teachman, 1987; Alexander and Eckland, 1974), psychology (Göllner et al., 2018; Guay et al., 2004; Flouri, 2006; Belsky et al., 2016; Boyle et al., 2007), and public health (Victora et al., 2015; Mojtabai et al., 2015).

Our key concern is how to model such an outcome. Consider Figure 1 which shows histograms of the years of education in several datasets (Current Population Survey, Flood et al. (2020); General Social Survey, Davis and Smith (1991); Add Health, Harris et al. (2019); \& Health and Retirement Study (HRS), Sonnega et al. (2014)). These distributions show a "clumping" of values that reflects the fact that people tend to leave school at specified end-points; in particular, note the cluster at 12 and 16 years reflecting completion of high school and 4-year college, respectively. Years of schooling from these studies are clearly not normally distributed. One potential approach for dealing with this outcome - the linear model - does not require that they are, only that they be conditionally normal. However, we argue that it is inappropriate to suppose that they are conditionally normal for most reasonable conditioning variables.

[Figure 1 about here.] 
Further, there are reasons to suspect that treating the scale as interval-i.e., differences across the scale are consistently meaningful in terms of representing equal changes in some base unit (Michell, 1986) - may distort subsequent inference. That is, the meaning entailed by a one-year increase in years of schooling may vary across regions of the educational attainment distribution. Although this scale is an interval scale of time, it may not be "interval" in its association with other variables. ${ }^{1}$ For example, an extra year of high school is quite inexpensive both in terms of out-of-pocket (i.e., there is no tuition at public schools) and opportunity costs while an extra year of college may be quite expensive in terms of out-of-pocket costs. Or, going from 11 to 12 years of schooling may be associated with a decrease in some disease later in life while there may be no predicted change associated with going from 12 to 13 years. Application of the linear model does not readily allow for us to identify such variation.

Given these facts, we ask: What alternatives to the standard linear model exist for analyzing educational attainment? One good option is to focus on the fact that the levels of educational attainment are well-ordered and to use ordinal models. Several techniques exist for analysis of ordinal outcomes (examples include: Lin (2020); Cameron and Heckman (2001); Van Ours and Veenman (2006)). We consider an approach-the cumulative link model Christensen (2018) - that has many desirable features (and that has key similarities to many previously used approaches). Via simulation, we (i) show the cumulative link model (CLM) behaves appropriately when the linear model is in fact true, (ii) that we can empirically test between whether the linear or CLM in fact generated the data, and (iii) show that the linear model may produce suboptimal (in terms of bias or precision, depending on the circumstances) estimates when the CLM is in fact true. These simulations offer guidance on specific instances in which the linear model is especially likely to be ill-suited for parameter estimation. We then illustrate the potential benefits of from fitting the ordinal model to empirical data using examples from the HRS.

\section{Contrasting Conceptual Models}

We begin with a discussion of the key conceptual components that we will consider below. These componentsall based on differences in how the expectation of some outcome $y$ varies as a function of a predictor $x$ - are shown in Figure 2. Here, we are discussing the true association between $x$ and $y$; following this, we turn to a discussion of how to model these differences. The top panel begins with a conventional scenario wherein $\mathbb{E}(y)$ is a linear function of $x$. In this case, the linear model is clearly the appropriate choice for understanding variation in $y$ as a function of $x$.

[Figure 2 about here.] 
Suppose that we do not observe such continuous variation in $\mathbb{E}(y)$. Instead, as in the second panel, consider a scenario wherein $y$ is only observed at discrete (rather than continuously-varying) values. In this case, a linear approximation will be appropriate especially if the discrete elements of $y$ are relatively close (so that the individual "steps" in $\mathbb{E}(y)$ are not "too big"). If there is some latent variable $y^{\prime}$ - wherein (i) $y$ is merely a coarsened (so that it only takes integral values) version of $y^{\prime}$ and (ii) $y^{\prime}$ is a linear function of $x$-then this second panel would be the result. Moving to the third panel, we now observe variation in the size of the horizontal steps (the vertical steps are still homogeneous). In the terminology we introduce below, the underlying steps in $x$ resulting in changes in $\mathbb{E}(y)$ are not "equidistant".

The question of interest here is how to best model coarsened variables $y$. Our argument is that when confronted with a coarsened variable $y$ (as in the second and third panels), one should start with the relatively conservative ordinal approach. If $y$ is merely a coarsened version of some latent variable $y^{\prime}$ that is, in fact, a linear function of $x$, the ordinal approach will produce estimates on par with those derived from the linear model. Moreover, estimates from the ordinal approach can be tested to determine whether the lineal model is a reasonable assumption. If $y$ is produced by something more nearly resembling the third panel, estimates from the linear model may be imprecise in some cases and highly biased in others. Thus, a treatment of the differences in $\mathbb{E}(y)$ as ordinal in the last two panels may allow for improved understanding of the relationship between $x$ and $y$ at little to no cost.

\subsection{Formal Models}

We now introduce the relevant formal models underlying the issues shown in Figure 2. Throughout this discussion and the paper, we distinguish between the data-generating model (DGM) and the data-analytic model (DAM). The models we introduce may act as either DGM and DAM. We focus on the simple case of a single predictor $x$.

Cumulative link model (CLM). We begin with a model for ordinal outcomes. The CLM (Christensen, 2018), treats $y_{i}$ as an ordinal variable rather than an intervally-scaled continuous variable. ${ }^{2}$ This model $^{2}$ asserts that

$$
\operatorname{Pr}\left(y_{i} \leq j \mid x_{i}\right)=F\left(\theta_{j}-\beta x_{i}\right)
$$

for each category $j$ into which $y$ might fall; for years of education, perhaps $j \in\{8, \ldots, 20\}$. Note that the actual values of $j$ are irrelevant and we will arbitrarily write $j \in\{1, \ldots, J\}$; in contrast, the value of $J$ will be crucial. The $F$ function is a link function; here, we assume that the link is the probit, i.e., $F\left(\theta_{j}-\beta x_{i}\right)=\Phi\left(\theta_{j}-\beta x_{i}\right)$ where $\Phi$ is the CDF for the standard normal. Other choices are possible; we focus on the probit given that it offers a straightforward method for comparing estimates from the CLM to linear alternatives. As one key 
point of intuition, if $y$ and $x$ are positively correlated, then we will observe $\beta>0$. That is, increased values of $x$ will effectively translate into downward shifts in the arguments of $F()$.

It is perhaps more straightforward to understand Eqn 1 in terms of the probabilities of responses in each category. Using the notion of a "category response function" from item response theory (Samejima, 1997), if we define the quantity in Eqn 1 as $\operatorname{Pr}\left(y_{i} \leq j \mid x_{i}\right)=\psi_{j}$ then we can write

$$
\operatorname{Pr}\left(y_{i}=j \mid x_{i}\right)=\psi_{j}-\psi_{j-1}
$$

Figure 3 Panel A considers such curves for a specific set of parameters. Note that, for a given value of $x$, we have $\sum_{j} \operatorname{Pr}\left(y_{i}=j \mid x_{i}\right)=1$ given that $y_{i}$ must be in one of the $j$ categories. For $j=1$ and $j=J$ (the boundary categories), the curves are monotonically decreasing and increasing respectively thus reflecting the fact that very small and very large values of $x$ are nearly certain to be in the first or last category respectively. For the middle categories, the curves represent small probabilities at the extremes with some global maxima. Figure 3 Panel B considers $\mathbb{E}(y)$ as a function of $x$ based on the category response probabilities ${ }^{3}$; note the similarity to the second panel of Figure 2.

[Figure 3 about here.]

Linear model (LM). So as to contrast some of its features with the CLM, we now consider the linear model (LM) for years of education, $y=\beta_{0}+\beta_{1} x_{i}$. For comparison to the CLM, we derive an expression similar to Eqn 2 for the LM case. The standard version of this model asserts that the probability density associated with an observation of $y_{i}$ is

$$
f\left(y_{i} \mid x_{i}\right)=\frac{1}{\sqrt{2 \pi \sigma}} \exp \left(-\frac{1}{2}\left(\frac{y_{i}-\left(\beta_{0}+\beta_{1} x_{i}\right)}{\sigma}\right)^{2}\right) .
$$

This is the standard linear model, $\mathbb{E}\left(y_{i} \mid x_{i}\right)=\beta_{0}+\beta x_{i}$, supplemented with the assumptions that errors are homoscedastic. We can then compute

$$
\operatorname{Pr}\left(y \leq j \mid x_{i}\right)=\int_{-\infty}^{j} f\left(t \mid x_{i}\right) d t
$$

Using that, we derive

$$
\operatorname{Pr}\left(y_{i} \leq j \mid x_{i}\right)-\operatorname{Pr}\left(y_{i} \leq j-1 \mid x_{i}\right)=\int_{j-1}^{j} f\left(t \mid x_{i}\right) d t
$$

In a manner similar to that of Figure 3, we can compute these values; see Figure 4. These pseudo-category 
response functions ${ }^{4}$ are similar to those in Figure 3, a function of the fact that both models rely on the CDF of the normal distribution and the fact that the $\theta$ values are evenly spaced in Figure 3 . The key difference is that here there are not category response functions for the top and bottom values as the DGM here leads to unbounded outcome values. While this is an important difference in the abstract, it may have less impact in real-world settings given that outcomes are frequently measured on scales with boundaries in practice.

[Figure 4 about here.]

Extensions of the CLM. We now consider two extensions of the CLM. The first pertains to a special property of CLM shown in Figure 3: The $\theta$ values are evenly spaced (i.e., differences between consecutive values are equivalent). We now further discuss the role of these parameters. The $\theta$ values are frequently referred to as "thresholds". We emphasize their role in Figure 3 by changing the background shading at these values. If $F(0)=0.5$ (which is true for the probit link), then $\theta_{j}$ reflects the value at which a value of $x \beta$ has even odds of being in category $j$ or below versus category $j+1$ or above. Note that the differences in color do not perfectly correspond to points where the category response curves cross. This is due to the fact that the thresholds are relative to statements about where there are even odds for the cumulative probabilities not the category probabilities.

The thresholds need not be evenly distributed as they are in Figure 3. We illustrate the case of nonuniform thresholds in Figure 5. Here, values of $x \in[-2,0.28]$ correspond to an expected value of 1 ; in contrast, there is a much smaller interval corresponding to $\mathbb{E}(y)=2$ and an even smaller interval corresponding to $\mathbb{E}(y)=3$. Thus, we can resolve the differences between panels 2 and 3 in Figure 2 into a question about the values of $\theta$. In particular, we can test if they are equidistant; we refer to p-values from such a test as $p_{\theta}$. Such tests will be illustrative in helping to determine whether the CLM or LM would be most appropriately used in a given case.

[Figure 5 about here.]

The second extension of the CLM is a way of allowing for flexibility into the CLM. In particular, note that Eqn 1 posits a uniform shift as a function of the covariate. ${ }^{5}$ This need not be the case. We can relax this assumptions by allowing for "nominal effects" wherein the effect of a covariate varies across the categories. This model - the LCM with nominal effects (CLMn) - asserts that

$$
\operatorname{Pr}\left(y_{i} \leq j \mid x_{i}\right)=F\left(\theta_{j}-\beta_{j} x_{i}\right) .
$$

In this model, the distances between thresholds effectively vary as a function of $x$ (see Figure 2 in Christensen (2018)). As we discuss below in the context of an empirical example, this extension allows for a variety of 
empirical features of our data to become apparent.

\section{Simulation Studies}

In the below simulation studies, we consider estimates generated via LM and CLM as DAM for different choices of the DGM. In particular, we consider the sequence of studies suggested in Table 1. We first use the LM as the DGM and show that the CLM acts functionally equivalently to the LM for a DAM. We then turn to the CLM as DGM and illustrate shortcomings of the LM when it is used as the DAM. Estimation of CLM is performed via Christensen (2019).

[Table 1 about here.]

\subsection{When LM is the DGM, the CLM is an efficient alternative.}

We simulate data as $y_{i}^{\star} \sim \mathrm{N}\left(\beta x_{i}, \sigma^{2}\right)$ where $\beta \sim \operatorname{Unif}(0,2), x \sim \mathrm{N}(0,1)$, and $\sigma^{2} \in\{1,4,25\}$ and $1 \leq i \leq N$. We then round $y_{i}^{\star}$ to the nearest integer, this is $y_{i}$. Using $y_{i}$, we then estimate the LM and CLM; a comparison of point estimates relative to the true $\beta$ values is in Figure 6 for a subset of the generating conditions. Both sets of estimates are relatively accurate (i.e., they track the 45 degree line). The CLM estimates have a slightly smaller correlation with the true values as compared to the LM estimates, but the difference is indeed quite small.

[Figure 6 about here.]

This evidence suggests that the CLM can be used in most situations without a severe reduction in estimation quality (this is even true for smaller sample sizes, see Table 2). To account for uncertainty in estimates, we also compare $t$ statistics from LM and CLM estimates. When $\sigma^{2}$ is small, CLM test statistics are roughly $80 \%$ the size of their LM peers; differences decrease as $\sigma^{2}$ increases. Thus, estimates are nearly as precise when using CLM as LM and there is relatively little increase in uncertainty around these estimates.

[Table 2 about here.]

Further, we argue that any inadequacies of using the CLM as DAM when the LM is in fact the DGM can be mitigated by being able to detect the appropriate DGM (i.e., the LM). To do this, we can examine the $\theta$ values. In particular, the CLM can be used to adjudicate whether the LM is an appropriate model in a given scenario a test of whether such thresholds are equidistant. Under the LM, they should be given that

$$
\operatorname{Pr}\left(y_{i} \leq j\right)-\operatorname{Pr}\left(y_{i} \leq j-1\right)=\operatorname{Pr}\left(y_{i} \leq j-1\right)-\operatorname{Pr}\left(y_{i} \leq j-2\right)
$$


for all values of $j$. In the final column of Table 2, we show the proportion of tests of equidistant thresholds that would be rejected when the LM is the DGM. Values are very near the alpha level (0.05) for modestly sized samples suggesting that this test can be used to reliably identify cases wherein thresholds are equidistant and, consequently, the LM can be used as DAM.

\subsection{When the CLM is the DGM, LM-based estimates are less accurate.}

In contrast, when the LM is not the DGM, estimates derived from the LM can be suboptimal. We illustrate this by simulating data from the CLM where $\beta \sim \operatorname{Unif}[0,1], J \in\{5,10,20\}$, and values of $\theta_{j}$ are manipulated via two parameters, $L$ and $s$. The first parameter controls the range over which the non-boundary categories predominate. The second parameter controls the level of regularity in the sequence of thresholds; for $s=1$, the thresholds are evenly spaced. Details are as follows:

- For a given $J$, we first create a lattice of $s(J-1)$ evenly spaced $\theta_{j}$ values between $-L$ and $L$. Denote these points as $l=\left\{l_{1}, \ldots, l_{s(J-1)}\right\}$ (and note that $l_{1}=-L$ and $\left.l_{s(J-1}\right)=L$ by design).

- Create a subset of lattice points, $l^{\prime}$ by removing $l_{1}$ and $l_{s(J-1)}$.

- From the remaining lattice points in $l^{\prime}=\left\{l_{2}, \ldots, l_{s(J-1)-1}\right\}$, we sample $J-3$ values. Define this as $l_{\text {sample. }}$ Note that when $s=1, l^{\prime}=l_{\text {sample. }}$.

- Define the set of $\theta$ values as the set $\left\{l_{1}, l_{\text {sample }}, l_{s(J-1)}\right\}$.

A schematic comparing a hypothetical $l$ and $\theta$ for $J=10$ and $s=2$ is shown in Figure 7 . Note that higher values of $s$ lead to sets of $\theta$ values that are increasingly sparse subsets of $l$; that is, Figure 3 would correspond to $s=1$ whereas Figure 5 is consistent with $s>1$.

[Figure 7 about here.]

Results for simulations based on 1000 iterations for each configuration of parameters are in Table 3. Estimates from the CLM are generally more highly correlated with the true parameters than are estimates from LM although differences are relatively small for larger $N$. However, even for larger $N$, estimates from LM can be substantially noisier if either $L$ or $s$ is relatively large especially for small $J$. When $N=1000$ and $J=5$, LM-based estimates are relatively weakly correlated, $r=0.66$, with true parameters when $L=4$ and $s=3$ whereas the CLM-based estimates are highly correlated with true parameters, $r=0.97$. Even when $J=10$, estimates from LM have an appreciably larger amount of noise when $L=4$ and $s=3$.

[Table 3 about here.] 
To illustrate an even more concerning shortcoming of the LM when the CLM is the DGM, we also briefly consider interactions. We use Eqn 1 but use $\theta_{j}-\left(\beta_{1} x_{i}+\beta_{2} z_{i}+\beta_{3} x_{i} z_{i}\right)$ as the argument to $F()$ where $x$ and $z$ are simulated to be bivariate normal with unit variances and correlation of 0.4. We independently sample $\beta_{1}$ and $\beta_{2}$ from $\operatorname{Unif}(0,1)$ and set $\beta_{3}=0$. We then use both the LM and CLM as DAM and allow for estimates of $\beta_{3}$. We focus on the level of false discovery by testing whether estimates of $\beta_{3}$ are significant using $\alpha=0.05$. Results are shown in Table 4. Note that false discovery is appropriate (i.e., near 0.05) when $s=1$ both both the LM and CLM. The FDR is appropriate for all configurations of the parameters when the CLM is used as DAM. However, FDR is quite high when $s>1$ for the LM; this problem is more pronounced when $J$ is relatively small. This suggests that when the $\theta_{j}$ values are not evenly spaced, inferences regarding interactions from the LM will tend to produce significant interaction coefficients even when there is in fact no interaction.

[Table 4 about here.]

\subsection{Summary of Simulation Studies}

Results from simulation suggest that if the LM is the DGM, then there is little to lose if we use the CLM as the DAM. Estimates from CLM are nearly as efficient as those from the LM. Further, we can determine whether the LM is appropriate by examining the CLM thresholds. In contrast, if the CLM is the DGM, estimates from the LM are less precise. The differences in many cases are modest but can be more pronounced in cases with either relatively small $J$ or highly varying $\theta$. Further, inappropriate use of the LM when we attempted to model an interaction (which did not actually exist) led to high levels of false discovery. Collectively, this evidence suggests that ordinal approaches can be considered in at least complementary roles (if not outright substitutes) alongside traditional approaches for analysis of potentially ordinal variables such as years of education.

We further illustrate the benefits of the ordinal approach below in empirical analysis. We demonstrate two key facts. We first show that thresholds are not equidistant which thus suggests, based on simulation evidence, that the CLM may produce more accurate estimates. We then use the CLMn to emphasize that the homogeneity assumption of the linear model is often suboptimal and that more precise inferences may be obtained from the CLMn approach. 


\section{Empirical Illustration}

We now illustrate the potential benefits derived from the CLM approach using data from the HRS (Sonnega et al., 2014). The HRS is a biannual survey of Americans over 50 and their spouses; it is widely used to study the health and economic wellbeing of older Americans. This study asks about the number of years the respondent spent in school. ${ }^{6}$

We consider three predictors of years of education: father's not having a HS diploma, the respondent being female, and an indicator of the respondent's race and ethnicity (i.e., the respondent not being white or Hispanic). In the sample, $64 \%$ of respondents' fathers did not have a HS diploma, $56 \%$ were female, and $35 \%$ were non-white. Correlations between years of education and these variables are $-0.37,-0.01$, and -0.23 respectively. These correlations would be the effective outcomes of an approach based on LM; we now illustrate some additional insights that are easily derived using the ordinal approach.

We first estimate the CLM model in Figure 8. Consider the threshold estimates (i.e,. $\theta_{j}$ ), shown as vertical gray lines. Note first the fact that, in all cases, the thresholds are not equidistant (this is visually apparent; all $\left.p_{\theta}<1^{-10}\right)$. In all cases, the largest gap in the $\theta$ values occurs for the threshold between 12 and 13 years of education (i.e., no postsecondary schooling versus any attendance of postsecondary schooling). The non-equidistant thresholds suggest that the LM is not the DGM and thus the LM will not perform as well in terms of precise parameter recovery as will the CLM. The estimate of $\beta$ from Eqn 1 is emphasized as a red arrow (note that the arrow points from 0 to $-\beta$ so as to emphasize the shifts in intercepts in the appropriate direction). All estimates are significant and suggest rightward shifts in the intercepts; going from a 0 to 1 on any predictor would effectively move the thresholds up by a uniform amount with the effect of increasing the proportion of the distribution at lower levels of education.

[Figure 8 about here.]

We now consider CLMn estimates, see Figure 9. The estimate from the CLM model - the red arrow in Figure 8-is shown as a vertical red line. Heterogeneity in the effect at the different steps - i.e., the $\beta_{j}$ estimates in Eqn 6-are shown by the blue segments (which represent confidence intervals for the blue points). A father's not having a high school degree is strongest as a predictor for transitions between years of secondary school (i.e., below the lower gray line). Note that the relative decline in predictive power of father's not having a diploma for transitions following high school as opposed to the transition from 11 to 12 years of schooling echo those found in previous analysis (Mare, 1980).

[Figure 9 about here.] 
For being female, transitions at lower grades are below the mean effect and, in fact, below zero. This suggests a decrease in the thresholds for females. In contrast, at the higher levels, thresholds for females are relatively increased. Consequently, the distribution of the females' years of education is not well-characterized as a uniform shift of the males'. Rather, the female distribution has less variation than the male distribution; respondents with relatively little education are more likely to have a year or so more if they are female whereas relatively well-educated respondents are more likely to have a year or two less if female.

For nonwhite respondents, the effect of being nonwhite is a non-uniform but consistently negative shift in the offsets. The specific patterning of these results suggests that nonwhite respondents are much more likely to have fewer years of education but that being nonwhite being an increasingly muted predictor of educational differences amongst the well-educated. ${ }^{7}$

\section{Discussion}

Educational attainment is both a conceptually important variable and one that is easily and consistently measured in many kinds of surveys. Thus, it is widely used as an outcome. We present evidence to suggest that modeling it as an ordinal outcome may yield benefits. If a linear model is the DGM, the ordinal approach will be essentially equivalent in terms of resulting inferences and can also be used to test whether the interval-based LM approach is appropriate by testing whether thresholds are equidistant. If not, we show that there are benefits - both statistical and conceptual-to be had from application of the cumulative link model. The approach that we advocate could also be used to study alternative approaches to educational attainment (e.g., the same approach could be used if a person's highest educational credential is the outcome of interest).

Two findings from our simulation studies are particularly noteworthy. First, tests of $p_{\theta}$ are valuable. They can indicate when the LM is suitable as the $\theta$ values are relatively equidistant. When they are not, analysis via the LM is likely to be less precise than analysis via the CLM (especially when the number of categories, $J$, is relatively small). Second, when interactions are of interest, ordinal models are highly desirable (some studies are beginning to use such models (Lin, 2020)). A reliance on linear models in such cases can lead to high levels of false discovery.

We demonstrate the efficacy of our CLM approach using data from the HRS (Sonnega et al., 2014). Although we considered relatively straightforward cases, the evidence presented suggests that CLM estimates would be more precise than LM estimates. Furthermore, the CLMn approach is able to identify nuanced higher-order effects that offer us increased insight about the associations in question. In particular, we are able to readily detect that the main difference between the male and female distributions of years of education 
is in the second moment and that the difference between years of education amongst white and nonwhite respondents is most pronounced at lower-levels of education. There are, of course, other ways of noting these facts. However, we emphasize that these sets of findings both follow directly from a single analytic approach.

In the future, we would advocate for the following workflow when researchers consider educational attainment as an outcome:

- Fit CLMn. Examine the nominal effects.

- If the nominal effects show substantial variation, use this saturated model

- If not, go to:

- Fit CLM. Examine the thresholds.

- If thresholds vary, use this model.

- If thresholds are equidistant, go to:

- Utilize LM.

We think that this strategy for modeling educational attainment is fairly straightforward and easy to implement.

Future work may consider other issues; we emphasize two possibilities here. First, other variables should perhaps be treated similarly. One potential class of outcomes that may merit such treatment is sum scores (e.g., composites created by summing over positive responses to survey items). Such scores have even less claim to being interval (Domingue, 2014) and are thus even more problematic from the perspective of handling them as continuous outcomes. The ordinal approach we advocate for here may yield benefits when analyzing such variables. One outcome in particular that may merit such treatment is depression which is frequently modeled as such a sum score. Previous work has suggested that such modeling may be ineffective due to indicator-specific effects (Fried and Nesse, 2015) but we also note that such an outcome, if modeled using the LM, may also be suboptimal from a statistical perspective. Second, the cumulative link model offers additional flexibility that we do not discuss here. In particular, incorporation of scale effects (Christensen, 2018) may allow for identification of additional higher-order effects that would further illuminate mechanisms of relevance for the study of educational attainment. 


\section{Notes}

${ }^{1}$ In a technical sense, the issue here is not that the scale is non-interval but that associations are nonlinear (for example, see Montez et al. (2012).

${ }^{2}$ The CLM is also known as the ordered probit model when the normal CDF is used as the link function (Long and Freese, 2006).

${ }^{3}$ What is the smallest $j$ such that $\sum_{i \leq j} \psi_{i}>0.5$ ?

${ }^{4}$ Because $y_{i}$ here is not integral-valued.

${ }^{5}$ This phenomenon pertains to the "proportional odds" assumption that the effect of $x$ is constant across levels of $y$ in the case of the logit link. Given our focus on the probit link, we do not use this language.

${ }^{6}$ See the raedyrs variable, https://www.rand.org/content/dam/rand/www/external/labor/aging/dataprod/randhrs1992_ 2016v2.pdf.

${ }^{7}$ An alternative representation of these stylized facts could be framed via quantile regression (Koenker and Hallock, 2001). If $Q_{\tau}(Y \mid X)=\beta_{\tau}$ indicates an estimated association of $\beta_{\tau}$ between an outcome $Y$ and a predictor $X$ at the $\tau$-th quantile of $Y$, then: for female, $\beta_{\tau}$ would be positive for small $\tau$ and negative for large $\tau$; for nonwhite respondents, $\beta_{\tau}$ would decline as a function of $\tau$. 


\section{References}

Alexander, K. L. and B. K. Eckland (1974). Sex differences in the educational attainment process. American Sociological Review, 668-682.

Belsky, D. W., T. E. Moffitt, D. L. Corcoran, B. Domingue, H. Harrington, S. Hogan, R. Houts, S. Ramrakha, K. Sugden, B. S. Williams, et al. (2016). The genetics of success: How single-nucleotide polymorphisms associated with educational attainment relate to life-course development. Psychological science 27(7), 957-972.

Boyle, M. H., K. Georgiades, Y. Racine, and C. Mustard (2007). Neighborhood and family influences on educational attainment: Results from the ontario child health study follow-up 2001. Child development 78(1), $168-189$.

Cameron, S. V. and J. J. Heckman (2001). The dynamics of educational attainment for black, hispanic, and white males. Journal of political Economy 109(3), 455-499.

Christensen, R. H. B. (2018). Cumulative link models for ordinal regression with the $\mathrm{r}$ package ordinal. Journal of Statistical Software.

Christensen, R. H. B. (2019). ordinal-regression models for ordinal data. R package version 2019.12-10. https://CRAN.R-project.org/package=ordinal.

Conley, D., B. W. Domingue, D. Cesarini, C. Dawes, C. A. Rietveld, and J. D. Boardman (2015). Is the effect of parental education on offspring biased or moderated by genotype? Sociological science 2,82 .

Darnell, A. and D. E. Sherkat (1997). The impact of protestant fundamentalism on educational attainment. American Sociological Review, 306-315.

Davis, J. A. and T. W. Smith (1991). The NORC general social survey: A user's guide, Volume 1. SAGE publications.

Domingue, B. (2014). Evaluating the equal-interval hypothesis with test score scales. Psychometrika 79(1), $1-19$.

Flood, S., M. King, R. Rodgers, S. Ruggles, and J. Warren (2020). Integrated public use microdata series, current population survey: Version 8.0 [dataset].

Flouri, E. (2006). Parental interest in children's education, children's self-esteem and locus of control, and later educational attainment: Twenty-six year follow-up of the 1970 british birth cohort. British journal of Educational psychology 76(1), 41-55. 
Fried, E. I. and R. M. Nesse (2015). Depression sum-scores don`t add up: why analyzing specific depression symptoms is essential. BMC medicine 13(1), 1-11.

Göllner, R., R. I. Damian, B. Nagengast, B. W. Roberts, and U. Trautwein (2018). It's not only who you are but who you are with: High school composition and individuals' attainment over the life course. Psychological science 29(11), 1785-1796.

Guay, F., S. Larose, and M. Boivin (2004). Academic self-concept and educational attainment level: A ten-year longitudinal study. Self and identity 3(1), 53-68.

Harris, K. M., C. T. Halpern, E. A. Whitsel, J. M. Hussey, L. A. Killeya-Jones, J. Tabor, and S. C. Dean (2019). Cohort profile: The national longitudinal study of adolescent to adult health (add health). International Journal of Epidemiology 48(5), 1415-1415k.

Herd, P., J. Freese, K. Sicinski, B. W. Domingue, K. Mullan Harris, C. Wei, and R. M. Hauser (2019). Genes, gender inequality, and educational attainment. American Sociological Review 84(6), 1069-1098.

Koenker, R. and K. F. Hallock (2001). Quantile regression. Journal of economic perspectives 15(4), 143-156.

Lin, M.-J. (2020). The social and genetic inheritance of educational attainment: Genes, parental education, and educational expansion. Social science research 86, 102387.

Long, J. S. and J. Freese (2006). Regression models for categorical dependent variables using Stata, Volume 7. Stata press.

Mare, R. D. (1980). Social background and school continuation decisions. Journal of the american statistical association 75(370), 295-305.

Michell, J. (1986). Measurement scales and statistics: A clash of paradigms. Psychological bulletin 100(3), 398.

Mojtabai, R., E. A. Stuart, I. Hwang, W. W. Eaton, N. Sampson, and R. C. Kessler (2015). Long-term effects of mental disorders on educational attainment in the national comorbidity survey ten-year follow-up. Social psychiatry and psychiatric epidemiology 50(10), 1577-1591.

Montez, J. K., R. A. Hummer, and M. D. Hayward (2012). Educational attainment and adult mortality in the united states: A systematic analysis of functional form. Demography 49(1), 315-336.

Samejima, F. (1997). Graded response model. In Handbook of modern item response theory, pp. 85-100. Springer. 
Sonnega, A., J. D. Faul, M. B. Ofstedal, K. M. Langa, J. W. Phillips, and D. R. Weir (2014). Cohort profile: the health and retirement study (hrs). International journal of epidemiology 43(2), 576-585.

Teachman, J. D. (1987). Family background, educational resources, and educational attainment. American sociological review, 548-557.

Van Ours, J. C. and J. Veenman (2006). Age at immigration and educational attainment of young immigrants. Economics Letters 90(3), 310-316.

Victora, C. G., B. L. Horta, C. L. De Mola, L. Quevedo, R. T. Pinheiro, D. P. Gigante, H. Gonçalves, and F. C. Barros (2015). Association between breastfeeding and intelligence, educational attainment, and income at 30 years of age: a prospective birth cohort study from brazil. The lancet global health 3(4), e199-e205. 


\section{List of Figures}

1 Distribution of educational attainment in various data. . . . . . . . . . . . . . . . . 18

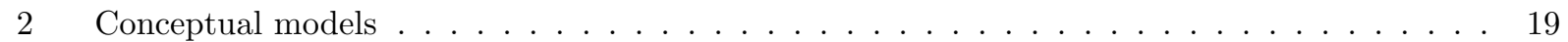

3 Illustration of CLM. Curves represent $\operatorname{Pr}(y=j \mid x)$ where $J=5, \beta=1, x \sim \mathrm{N}(0,1)$, and $\theta=\{-2,-0.67,0.67,2\}$. Shaded areas represent regions between different values of $\theta$. Color of curve (from blue to red) corresponds to increasing value of $j$. . . . . . . . . . . . . . 20

4 Illustration of pseudo-category response functions for LM (i.e., curves represent: $\operatorname{Pr}(y \leq$ $j \mid x)-\operatorname{Pr}(y \leq j-1 \mid x))$ where $\beta=1, x \sim \mathrm{N}(0,1)$ and Eqn 3 is the DGM. . . . . . . . . . 21

5 Illustration of CLM. Curves represent $\operatorname{Pr}(y=j \mid x)$ where $J=5, \beta=1, x \sim \mathrm{N}(0,1)$, and $\theta=\{-2,0,1,1.5\}$. Shaded areas represent regions between different values of $\theta$. . . . . . . 22

6 Scatterplot of true $\beta$ parameters versus LM and CLM estimates for $N=1000$ and $\sigma^{2}=1$. . 23

$7 \quad$ A schematic comparing $l$ and $\theta$ for $J=10$ and $s=2 \ldots \ldots$. . . . . . . . . . . . . . . 24

8 Estimates of $\theta$ and nominal offsets based on three models of educational attainment. . . . . . 25

9 Comparison of CLM and CLMn estimates from HRS data. Main effect estimates from CLM are shown as vertical red lines. Category-specific offsets are shown as blue points (plus 95\% confidence intervals). . . . . . . . . . . . . . . . . . . . . . . . . . . 26 
Figure 1: Distribution of educational attainment in various data.

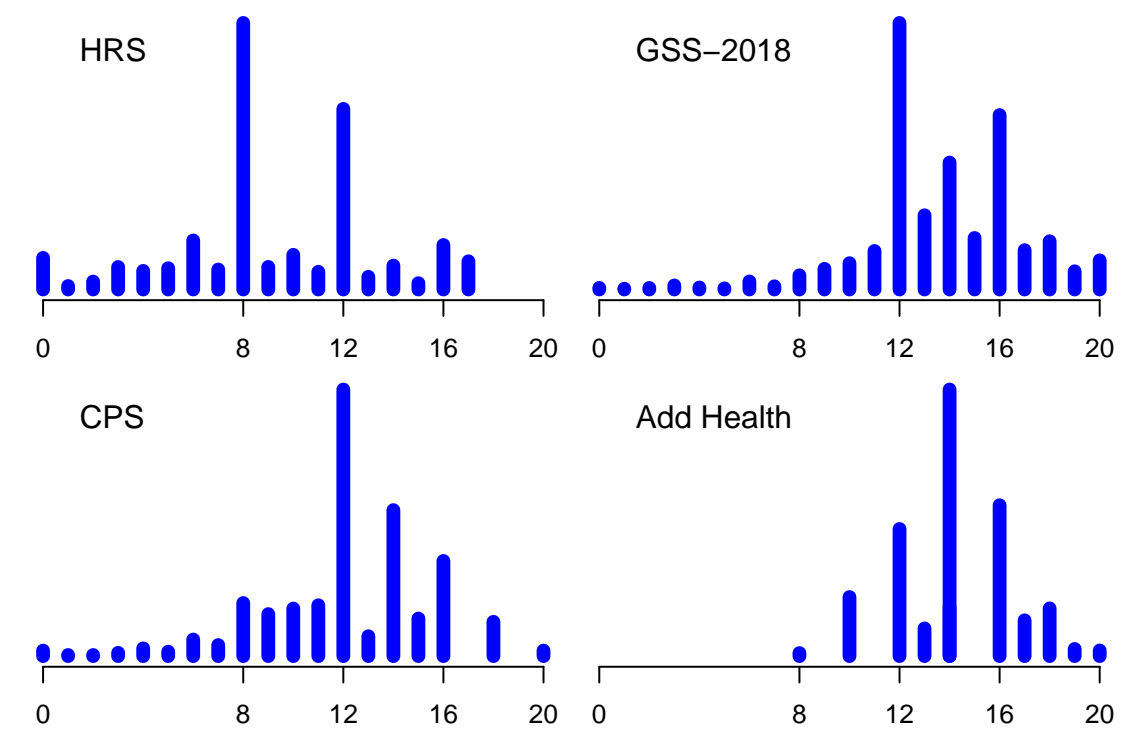


Figure 2: Conceptual models
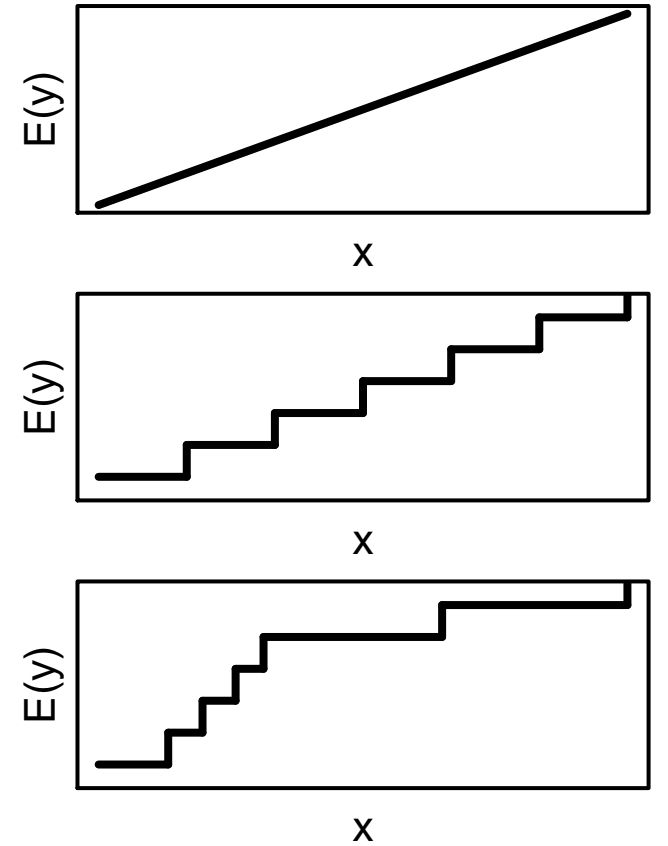
Figure 3: Illustration of CLM. Curves represent $\operatorname{Pr}(y=j \mid x)$ where $J=5, \beta=1, x \sim \mathrm{N}(0,1)$, and $\theta=$ $\{-2,-0.67,0.67,2\}$. Shaded areas represent regions between different values of $\theta$. Color of curve (from blue to red) corresponds to increasing value of $j$.
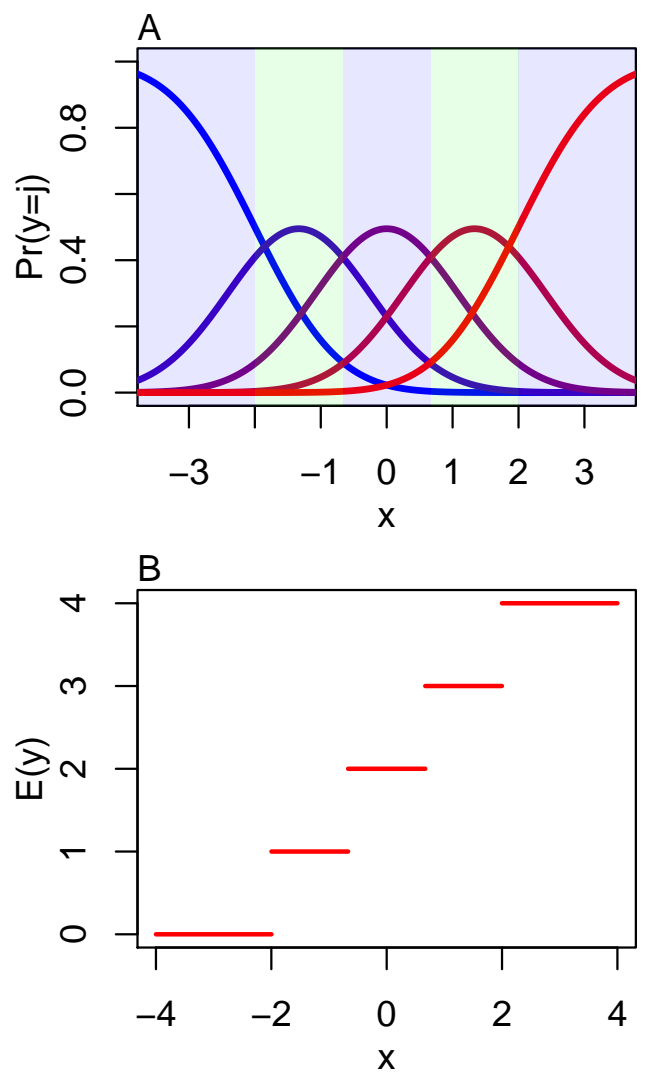
Figure 4: Illustration of pseudo-category response functions for LM (i.e., curves represent: $\operatorname{Pr}(y \leq j \mid x)-$ $\operatorname{Pr}(y \leq j-1 \mid x))$ where $\beta=1, x \sim \mathrm{N}(0,1)$ and Eqn 3 is the DGM.

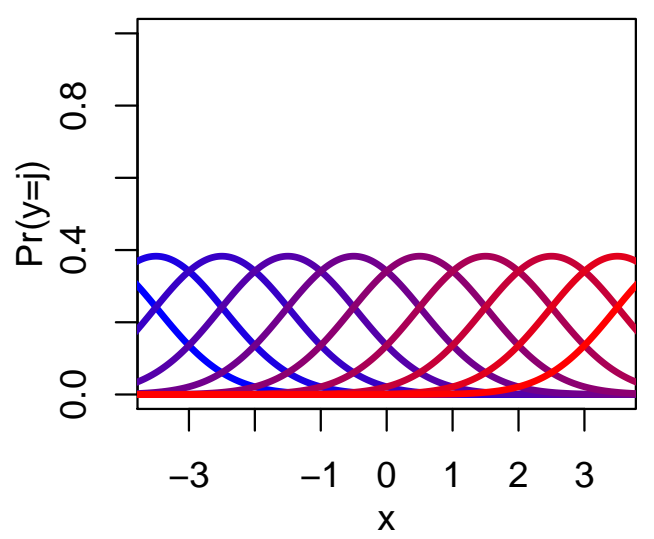


Figure 5: Illustration of CLM. Curves represent $\operatorname{Pr}(y=j \mid x)$ where $J=5, \beta=1, x \sim \mathrm{N}(0,1)$, and $\theta=$ $\{-2,0,1,1.5\}$. Shaded areas represent regions between different values of $\theta$.
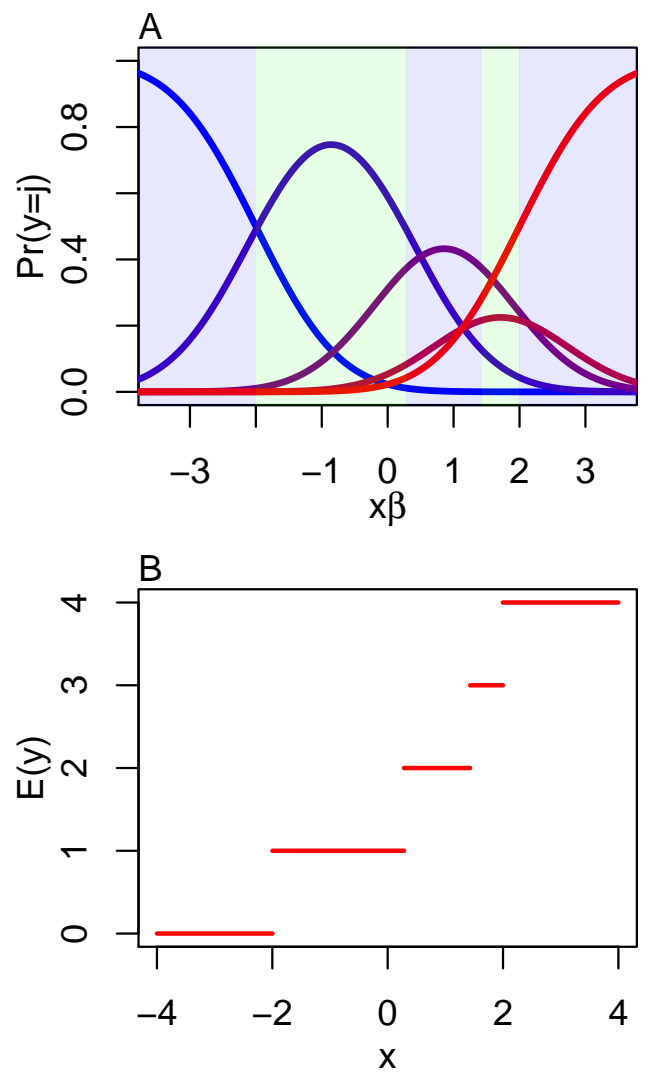
Figure 6: Scatterplot of true $\beta$ parameters versus LM and CLM estimates for $N=1000$ and $\sigma^{2}=1$.

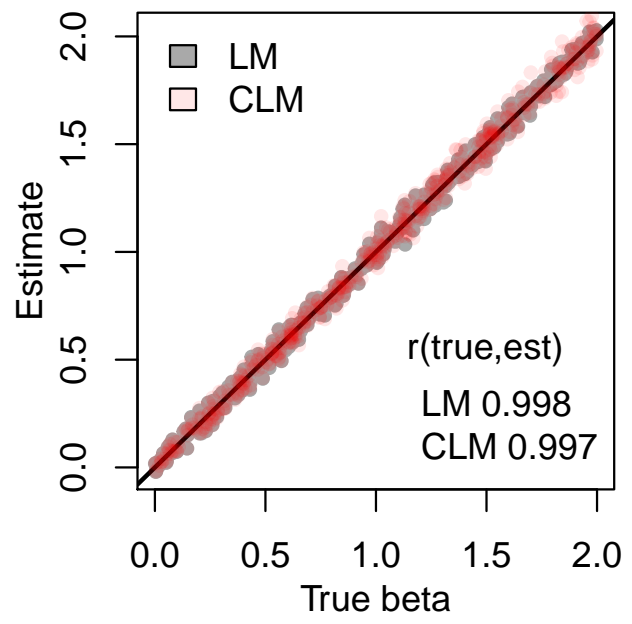


Figure 7: A schematic comparing $l$ and $\theta$ for $J=10$ and $s=2$.

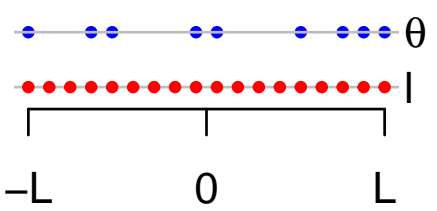


Figure 8: Estimates of $\theta$ and nominal offsets based on three models of educational attainment.
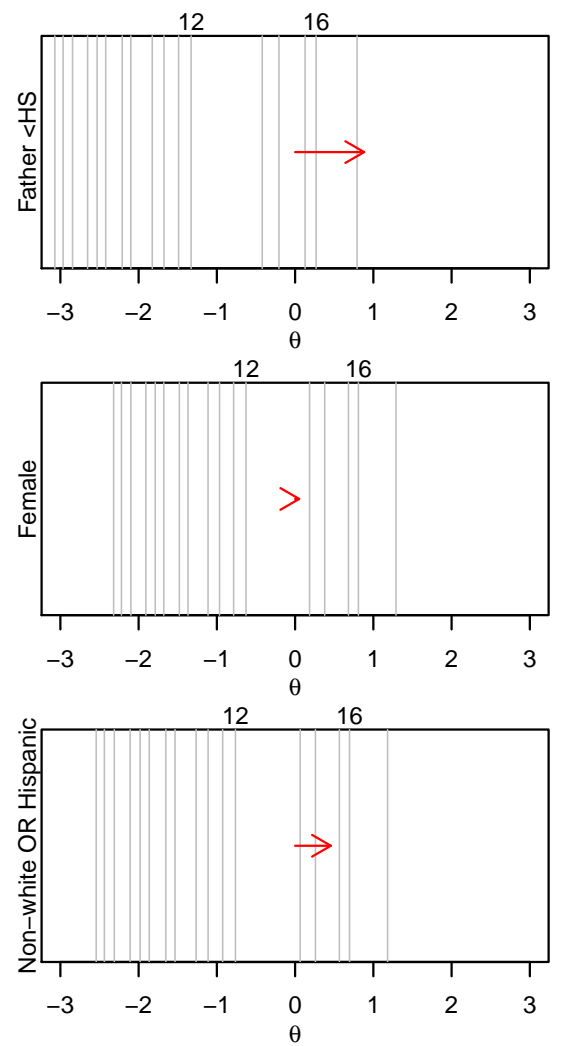
Figure 9: Comparison of CLM and CLMn estimates from HRS data. Main effect estimates from CLM are shown as vertical red lines. Category-specific offsets are shown as blue points (plus $95 \%$ confidence intervals).
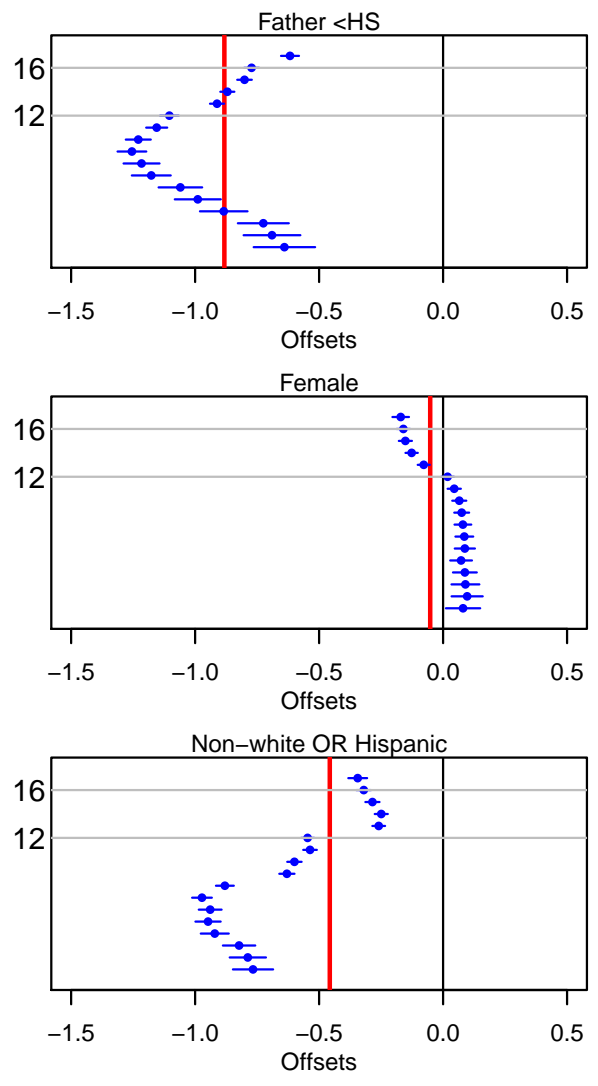


\section{List of Tables}

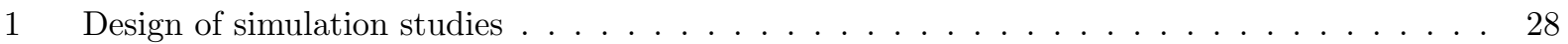

2 Correlations between true parameters and LM/CLM estimates (when LM is the DGM) for

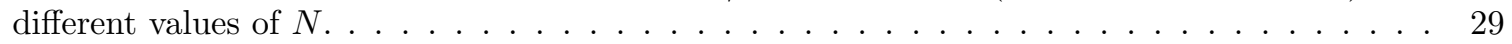

3 Correlations $(r)$ between LM and CLM estimates when the CLM is the DGM. $\ldots \ldots \ldots$

4 False discovery rate for interaction analyses $(N=1000, L=3) \ldots \ldots \ldots \ldots$ 
Table 1: Design of simulation studies

\begin{tabular}{lcc}
\hline & \multicolumn{2}{c}{ DAM } \\
\cline { 2 - 3 } DGM & LM & CLM \\
\hline LM & & 1 \\
CLM & 2 & \\
\hline
\end{tabular}


Table 2: Correlations between true parameters and LM/CLM estimates (when LM is the DGM) for different values of $N$.

\begin{tabular}{lcccccc}
\hline & & \multicolumn{2}{c}{$r$ (true, est) } & & \multicolumn{2}{c}{ Mean } \\
$\mathrm{N}$ & $\bar{J}$ & LM & CLM & & $\frac{t_{\text {CLM }}}{t_{\text {LM }}}$ & $p_{\theta}<.05$ \\
\hline$\sigma^{2}=1$ & & & & & \\
100 & 8 & 0.981 & 0.969 & & 0.798 & 0.040 \\
1000 & 10 & 0.998 & 0.997 & & 0.800 & 0.056 \\
10000 & 12 & 1.000 & 1.000 & & 0.800 & 0.038 \\
\hline$\sigma^{2}=4$ & & & & & \\
100 & 11 & 0.944 & 0.931 & & 0.878 & 0.028 \\
1000 & 15 & 0.994 & 0.993 & & 0.875 & 0.036 \\
10000 & 18 & 0.999 & 0.999 & & 0.929 & 0.044 \\
\hline$\sigma^{2}=25$ & & & & & \\
100 & 22 & 0.747 & 0.735 & & 0.992 & 0.002 \\
1000 & 32 & 0.967 & 0.966 & & 0.988 & 0.032 \\
10000 & 38 & 0.996 & 0.996 & & 0.987 & 0.034 \\
\hline
\end{tabular}


Table 3: Correlations $(r)$ between LM and CLM estimates when the CLM is the DGM.

\begin{tabular}{ccccc}
\hline & & & \multicolumn{2}{c}{$r$ (true, est) } \\
\cline { 4 - 5 }$J$ & $L$ & $s$ & LM & CLM \\
\hline$N=250$ & & & & \\
5 & 2 & 1 & 0.97 & 0.97 \\
5 & 2 & 3 & 0.93 & 0.96 \\
5 & 4 & 1 & 0.96 & 0.96 \\
5 & 4 & 3 & 0.66 & 0.90 \\
10 & 2 & 1 & 0.98 & 0.97 \\
10 & 2 & 3 & 0.96 & 0.97 \\
10 & 4 & 1 & 0.97 & 0.97 \\
10 & 4 & 3 & 0.84 & 0.96 \\
20 & 2 & 1 & 0.97 & 0.97 \\
20 & 2 & 3 & 0.97 & 0.97 \\
20 & 4 & 1 & 0.98 & 0.97 \\
20 & 4 & 3 & 0.92 & 0.97 \\
\hline$N=1000$ & & & & \\
5 & 2 & 1 & 0.99 & 0.99 \\
5 & 2 & 3 & 0.96 & 0.99 \\
5 & 4 & 1 & 0.99 & 0.99 \\
5 & 4 & 3 & 0.66 & 0.97 \\
10 & 2 & 1 & 0.99 & 0.99 \\
10 & 2 & 3 & 0.97 & 0.99 \\
10 & 4 & 1 & 0.99 & 0.99 \\
10 & 4 & 3 & 0.88 & 0.99 \\
20 & 2 & 1 & 0.99 & 0.99 \\
20 & 2 & 3 & 0.98 & 0.99 \\
20 & 4 & 1 & 0.99 & 0.99 \\
20 & 4 & 3 & 0.94 & 0.99 \\
\hline$N=10000$ & & & & \\
5 & 2 & 1 & 1.00 & 1.00 \\
5 & 2 & 3 & 0.96 & 1.00 \\
5 & 4 & 1 & 1.00 & 1.00 \\
5 & 4 & 3 & 0.70 & 1.00 \\
10 & 2 & 1 & 1.00 & 1.00 \\
10 & 2 & 3 & 0.98 & 1.00 \\
10 & 4 & 1 & 1.00 & 1.00 \\
10 & 4 & 3 & 0.88 & 1.00 \\
20 & 2 & 1 & 1.00 & 1.00 \\
20 & 2 & 3 & 0.99 & 1.00 \\
20 & 4 & 1 & 1.00 & 1.00 \\
20 & 4 & 3 & 0.94 & 1.00 \\
\hline & & & &
\end{tabular}


Table 4: False discovery rate for interaction analyses $(N=1000, L=3)$.

\begin{tabular}{cccc}
\hline & & \multicolumn{2}{c}{ FDR } \\
\cline { 3 - 4 }$J$ & $s$ & LM & CLM \\
\hline 5 & 1 & 0.044 & 0.053 \\
5 & 3 & 0.501 & 0.05 \\
5 & 5 & 0.503 & 0.049 \\
\hline 10 & 1 & 0.047 & 0.059 \\
10 & 3 & 0.408 & 0.052 \\
10 & 5 & 0.443 & 0.038 \\
\hline 25 & 1 & 0.041 & 0.052 \\
25 & 3 & 0.265 & 0.051 \\
25 & 5 & 0.27 & 0.062 \\
\hline
\end{tabular}

